\section{Unit Cell and Preliminary Structure for Cellulose Triacetate}

Although interplanar spacings for cellulose acetate II have been published ${ }^{1,2}$, I have been unable to trace a determination of the unit cell or space group.

Small films of highly crystalline triacetate with good fibrous orientation and a fair degree of biaxial orientation have been prepared by repeated rolling at successively higher temperatures between $160^{\circ}$ and $215^{\circ} \mathrm{C}$. The higher orientation was somewhat improved by swelling in glacial acetic acid, followed by drying at $110^{\circ} \mathrm{C}$. while maintaining the sample under pressure. Figs. $1 a$ and $b$ are $\mathrm{X}$-ray diffraction photographs of such films showing several new spacings. On the perpendicular photographs along the equator, there are reflexions with the spacings 24.50 and $12.25 \mathrm{~A}$., and on the first level there is a 9.61-A. spacing. On the parallel photograph there is a faint $5 \cdot 78$-A. spacing along the equator. It can be seen that the fibre orientation is better in a plane containing the fibre axis and a perpendicular to the plane of the sheet, than in the plane of the sheet.

The additional data have made it possible to index all spots on the basis of an orthogonal unit cell containing four cellobiose hexa-acetate residues. The dimensions of this unit cell, taking $[00 l]$ as the fibre direction, are $a=24.50 \mathrm{~A} ., b=11 \cdot 56 \mathrm{~A}$. and $c=$ $10.43 \mathrm{~A}$. The theoretical density is $1.30 \mathrm{gm}$./c.c., and this compares with $1.29 \mathrm{gm}$./c.c. observed for a fairly well-crystallized sample. Thirty reflexions have been indexed on this basis, and all have spacings in agreement with those calculated to within 1 per cent.

The orthogonal cell and the symmetry of the photographs suggest a space group of the orthorhombic system. The $(h 00),(0 k 0)$ and $(00 l)$ reflexions, except for a weak (100), are apparently absent for $h, k$ or $l$ odd. The presence of $(100)$ suggests the space group, $P 22_{1} 2_{1}$, if the screw axes in the $b$ - and $c$-directions are regarded as real. Other orthorhombic possibilities are $P 222, P 22_{1} 2$ and $P 222_{1}$. All these space groups have a two-fold axis in the $a$-direction, but it appears to be impossible to set up a model with two pairs of cellulose chains related by such a two-fold axis and which will give rise to a weak (020) reflexion. It was therefore decided that cellulose triacetate is only pseudo-orthorhombic and the probable space group is $P 2_{1}$, but that it may be closely approximated by $P 2_{1} 2_{1} 2_{1}$.
In the case of cellulose I and II, the cellulose chains are necessarily coincident with the $2_{1}$ axes, but this is not a requirement in this case. In one model based on $P 2_{1} 2_{1} 2_{1}$, which gives promising agreement with the intensities, the cellulose triacetate chains are paired about a $c_{2}$-axis, with the planes of the glucose residues parallel to one another and inclined at about $45^{\circ}$ with respect to $a$. The adjacent $c_{2}$-axes then have similar pairs of chains directed in the opposite direction and inclined at about $-45^{\circ}$.

The intensities are quite sensitive to the positions of the acetyl groups, and a refinement of this model promises considerable structural detail which will be published elsewhere.

Research Laboratories,

Eastman Kodak Company, Rochester 4, N.Y. Aug. 17.

1 Hess, K., and Trogus, C., Z. phys. Chem., B5, 161 (1929).

Baker, W. O., Fuller, C. S., and Pape, N. R., J. Amer. Chem. Soc., 6:, 3275 (1932).

\section{Production of Magnetic Fields in Liquid Mercury by Mechanical Motion}

IN connexion with recent attempts by Alfvén, Biermann, Batchelor et al., to explain stellar and interstellar magnetic fields, we have considered the possibility of producing electric currents and magnetic fields in liquid mercury by suitable mechanical motion.

Our experiments are arranged as follows. In $a_{\text {. }}$ cylindrical vessel filled with mercury we have rotated the upper half by means of a stirrer, while the lower half was prevented from rotation by means of radially fixed vanes on the bottom. The angular velocity of the mercury in the upper half was fairly high (about $20-35$ c./sec.) and slowed down to zero in the lower part of the vessel. Accordingly, there exists a rotational and toroidal flow of liquid mercury. The present research is restricted to the action of the toroidal flow only. In the absence of any outer magnetic field, the electrons appear to go somewhat ahead of the ions, so that there results a toroidal electric current causing a circular magnetic field around the axis of rotation. We have succeeded in detecting this magnetic field and find a value of $10^{-4}$ gauss, which seems to fit reasonably well with theoretical expectations.

It may seem difficult to screen off the magnetic field of the earth to less than $10^{-4}$ gauss over the whole area involved. Indeed, using large Helmholtz coils and enclosing the whole apparatus in a box of suitable screening material, there still remained a field of the strength of some $10^{-4}$ gauss. But fortunately the effect described above can be separated quite simply from the influence of the earth's magnetic field, as the former increases with the square of the speed of rotation, the latter linearly. Reversing the sense of rotation causes the two effects to be added or to be subtracted. This easily enables the evaluation of the magnetic field-strength created within the
Fig. 1. X-ray diffraction pattern of highly oriented crystalline cellulose triacetate. Copper $\boldsymbol{K} a$ monochromatic radiation. $(a)$ X-rays parallel to the plane of 